\title{
Hydrobiologia ogólna
}

A. LITYNSKI

Warszawa, Państwowe Wydawnictwo Naukowe, 1952, 545 p p., 277 figs., portrait de l'auteur (en langue polonaise).

Table des matières (pp. 21 - 506, chapitres I - X): Introduction. Hydrosphère comme milieu de vie des êtres vivants. Régions biologiques de l'eau. Organismes aquatiques et le milieu physique. Lumière comme facteur écologique. Bilan thérmique des eaux et son importance pour la vie des êtres vivants. Régime gazeux de l'hydrosphère. Relation entre les êtres vivants et les sels minéraux dissouts dans l'eau. Rôle des composants nutritifs dissouts dans l'eau pour les êtres aquatiques. Productivité biologique des eaux.

L'auteur de la note présente a fait précéder le texte proprement dit par une préface, un bref curriculum vitae et une bibliographie complète des travaux scientifiques de A. LITYNSKI. C'est par lui, que le livre complété par les données hydrobiologiques plus récentes et pourvu d'illustrations, a été préparé a la publication. Malgré ces suppléments la disposition primaire du matériel et les idées fondamentales de LITYŃSKI ont été conservées, sans changements. Le livre de A. LITYŃsKI est écrit avec beaucoup de talent. LITYŃsKi était pendant de longues années directeur de la Station Hydrobiologique du lac de WIGRY et en outre un savant plein de mérite, car il a contribué au développement des recherches hydrobiologiques en PoLOGNE. C'est une tentative hardie de réunir dans une seule oeuvre tous les problèmes hydrobiologiques fondamentaux concernant les eaux douces et salées. En comparant les résultats des recherches des deux branches essentielles d'hydrobiologie et notamment de limnologie et d'océanologie, l'auteur à maintes reprises arrive à des conclusions intéressantes et originales. Le livre contient un matériel, abondant résultat des recherches hydrobiologiques polonaises, mais comme on peut se rendre compte aisément en examinant la littérature, les idées et les considérations de l'auteur se basent sur les acquisitions mondiales dans le domaine de l'hydrobiologie.

Nous devons à l'effort créateur de LITYŃski l'originalité de cette œuvre hydrobiologique intéressante qui rendra un très grand service non seulement aux étudiants, mais aussi aux spécialistes.

L. K. PAWLOWSKI 